\title{
Crónica
}

\section{Revisión por pares, año 2003}

\author{
Peer-reviewers, year 2003
}

La Revista Médica de Chile emplea el sistema de evaluación por pares, independientes de los Editores, para seleccionar los artículos que publica y para estimular a los autores a perfeccionar sus manuscritos al mejor nivel que pueda alcanzarse en nuestro medio. A revisores externos se envían todos los artículos de investigación que pasaron el filtro de una primera revisión formal por los Editores, y la mayoría de los artículos de revisión y de presentación de casos clínicos. Los manuscritos de otra naturaleza son juzgados sólo por los Editores. En todas estas circunstancias, la decisión final de publicar un manuscrito recae en los Editores.

La aplicación de este sistema -desde la década de 1970- ha influido para que esta Revista difunda una alta proporción de los manuscritos generados en nuestro país, sobre temas de la clínica médica y de biomedicina. Es uno de los requisitos para incluirla en los Índices Bibliográficos de mayor importancia internacional, exigido también para pertenecer a organizaciones como la Asociación Mundial de Editores de Revistas Médicas (WAME).

Reconociendo la importancia que tiene para la Revista la colaboración desinteresada de sus revisores externos, todos los años se publica -en el número de julio- la nómina de quienes revisaron uno o más manuscritos durante el año calendario previo. En el año 2003 participaron en este proceso 342 personas, cuya nómina se publica a continuación. Al exponer sus nombres en un sitio destacado y cronológicamente estable de la Crónica, facilitamos a cada uno de ellos certificar ante cualquier institución, comité o persona, el reconocimiento oficial de la Revista Médica de Chile por su participación como Revisor Externo de manuscritos durante 2003. El rol del revisor externo, solicitado por los Editores de una revista, se considera una distinción y un reconocimiento de la capacidad e idoneidad de la persona invitada, quien participa en un proceso serio y responsable.

Llamamos la atención al aumento anual del número de revisores que han colaborado con la Revista: 155 en el 2000, 143 en 2001, 197 en 2002 y 341 en 2003 (crecimiento a $220 \%$ en los 4 años recientes). Ello ilustra el esfuerzo por perfeccionar la selección de los artículos que se publican, pero implica un recargo muy importante del trabajo de secretaría cuyas implicancias ya describimos en una Editorial reciente y está en conocimiento de la Presidencia de la Sociedad Médica de Santiago (Rev Med Chile 2004; 132: 7-10).

Agregamos en esta Crónica los nombres de personas que -por invitación de los Editoresrevisaron libros publicados por autores nacionales y comentaron sus características para facilitar su difusión entre sus potenciales lectores. Esta labor nos parece destacable por cuanto avala la importancia de dar a conocer la producción intelectualcientífica en nuestro ambiente.

A todos ellos expresamos el agradecimiento de los Editores de la Revista Médica de Chile, del Directorio de la Sociedad Médica de Santiago (Sociedad Chilena de Medicina Interna), de los autores de manuscritos publicados y de los lectores de la Revista. 
A

$\begin{array}{lll}\text { Abarca } & \text { V } & \text { Katia } \\ \text { Acuña } & \text { M } & \text { Raúl } \\ \text { Acuña } & \text { P } & \text { Mónica } \\ \text { Afani } & \text { S } & \text { Alejandro } \\ \text { Aguayo } & \text { B } & \text { Alberto } \\ \text { Alarcón } & \text { O } & \text { Teresa } \\ \text { Albala } & \text { B } & \text { Cecilia } \\ \text { Alvarez } & \text { U } & \text { Gonzalo } \\ \text { Alvo } & \text { A } & \text { Miriam } \\ \text { Aller } & \text { R } & \text { Felipe } \\ \text { Alliende } & \text { R } & \text { María Angélica } \\ \text { Amigo } & \text { C } & \text { Hugo } \\ \text { Ancic } & \text { C } & \text { Patricia } \\ \text { Apt } & \text { B } & \text { Werner } \\ \text { Araos } & \text { H } & \text { Daniel } \\ \text { Araya } & \text { Q } & \text { Verónica } \\ \text { Araya } & \text { D } & \text { Fernando } \\ \text { Arellano } & \text { H } & \text { Leonardo } \\ \text { Arrese } & \mathrm{J} & \text { Marco } \\ \text { Arteaga } & \text { U } & \text { Eugenio } \\ \text { Asenjo } & \text { M } & \text { Sylvia } \\ \text { Atalah } & \text { S } & \text { Eduardo } \\ \text { Atías } & \text { M } & \text { Antonio } \\ \text { Avendaño } & \text { C } & \text { Luis Fidel } \\ \text { Avendaño } & \text { B } & \text { Isabel } \\ & & \end{array}$

B

$\begin{array}{lll}\text { Badilla } & \text { O } & \text { Lautaro } \\ \text { Baeza } & \text { B } & \text { Ramón } \\ \text { Bannura } & \text { C } & \text { Guillermo } \\ \text { Barría } & \text { S } & \text { Manuel } \\ \text { Barriga } & \text { C } & \text { Francisco } \\ \text { Barros } & \text { R } & \text { Claudio } \\ \text { Barros } & \text { O } & \text { Felipe } \\ \text { Beca } & \text { I } & \text { Juan Pablo } \\ \text { Bedregal } & \text { G } & \text { Paula } \\ \text { Bello } & \text { S } & \text { Sergio } \\ \text { Berger } & \text { F } & \text { Zoltan } \\ \text { Bertin } & \text { C } & \text { Pablo } \\ \text { Besa } & \text { de C } & \text { Pelayo } \\ \text { Biagini } & \text { A } & \text { Leandro } \\ \text { Bianchi } & \text { Sch } & \text { Víctor } \\ \text { Bianchi } & \text { P } & \text { Marcelo } \\ \text { Bilbeny } & \text { L } & \text { Norberto } \\ \text { Blümel } & \text { M } & \text { Juan Enrique } \\ \text { Boehme } & \text { K } & \text { Cecilia } \\ \text { Borgoño } & \text { D } & \text { José Manuel } \\ \text { Braghetto } & \text { M } & \text { Italo } \\ \text { Brahm } & \text { B } & \text { Javier }\end{array}$

$\begin{array}{lll}\text { Bronfman } & \text { F } & \text { Lucía } \\ \text { Bunout } & \text { B } & \text { Daniel } \\ \text { Burrows } & \text { A } & \text { Raquel } \\ \text { Bustos } & \text { O } & \text { Eduardo }\end{array}$

C

Cabello

Cabrera

Cáceres

Calderón

Camacho

Canals

Cano

Capetillo

Cartier

Carvajal

Carvajal

Carvallo

Carvallo

Casar

Casas del Valle

Cassorla

Castillo

Castro

Cattani

Caviedes

Cifuentes

Contreras

Correa

Cortés

Corvalán

Cotera

Crosby

Cuchacovich

Cumsille

Chamorro

Chamorro

Chernilo

Chianale

Chomali

\section{D}

$\begin{array}{lll}\text { De Aretxabala } & \text { U } & \text { Xavier } \\ \text { De la Fuente } & \text { H } & \text { María } \\ \text { De la Maza } & \text { C } & \text { María Pía } \\ \text { De la Parra } & \text { C } & \text { Rodrigo } \\ \text { Díaz } & \text { J } & \text { Juan Carlos } \\ \text { Díaz } & \text { A } & \text { Patricia } \\ \text { Díaz } & \text { T } & \text { Violeta } \\ \text { Díaz } & \text { C } & \text { Jaime }\end{array}$




$\begin{array}{lll}\text { Dighero } & \text { T } & \text { Humberto } \\ \text { Domenech } & \text { L } & \text { Raúl } \\ \text { Domínguez } & \text { A } & \text { Raúl } \\ \text { Donoso } & \text { G } & \text { Verónica } \\ \text { Donoso } & \text { R } & \text { Rodrigo } \\ \text { D’Ottone } & \text { M } & \text { Karin } \\ \text { Duclos } & \text { H } & \text { Jaime } \\ \text { Durruty } & \text { A } & \text { Pilar } \\ & & \\ \text { E } & & \\ \text { Enríquez } & \text { L } & \text { Octavio } \\ \text { Espinoza } & \text { G } & \text { Ricardo } \\ \text { Eterovic } & \text { M } & \text { Ivo } \\ & & \\ \text { F } & & \\ \text { Fajuri } & \mathrm{N} & \text { Alejandro } \\ \text { Fasce } & \mathrm{H} & \text { Eduardo } \\ \text { Fernández } & \mathrm{V} & \text { Alejandra } \\ \text { Ferrés } & \mathrm{G} & \text { Marcela } \\ \text { Fielbaum } & \mathrm{C} & \text { Oscar } \\ \text { Figueroa } & \mathrm{G} & \text { Guillermo } \\ \text { Figueroa } & \mathrm{P} & \text { Jorge } \\ \text { Figueroa } & \mathrm{M} & \text { Claudia } \\ \text { Florenzano } & \mathrm{U} & \text { Fernando } \\ \text { Florenzano } & \mathrm{U} & \text { Ramón } \\ \text { Fodich } & \mathrm{F} & \text { Pedro } \\ \text { Fruns } & \mathrm{Q} & \text { Manuel } \\ \text { Fuenzalida } & \mathrm{P} & \text { Humberto } \\ \text { Fuster } & \mathrm{F} & \text { Francisco }\end{array}$

González

González

González

González

González

Gramegna

Guardia

Guglielmetti

Guiverneau

Gutiérrez

Guzmán

Guzmán

\section{H}

Hanne

Hering

Hermosilla

Herrera

Herrera

Herrera

Herrera

Herskovic

Hervé

Heusser

Hirsch

Hodgson

Honeyman

Horwitz

\section{I}

Ibarra

Inzunza

J

Jadue

Jalil

Jalil

Jalil

Jara

Jiménez

Jirón

Jofré

Juez

Juliet

K

Kauffmann

Koenig

Kottow
B Sergio

C Carlos

Y Mónica

D Robinson

O Ximena

S Gloria

B Silvia

$\mathrm{V}$ Antonio

B Mauricio

T Miguel

B Sergio

B Leonardo

A Christel

A Enrique

D Germán

L Renato

P Adela

M Mario

L Patricio

L Pedro

E Beatriz

$\mathrm{R}$ Felipe

B Sandra

B María Isabel

M Juan

C Nina

V Humberto

H Oscar

$\mathrm{H}$ Liliana

M Jorge

M Roberto

L Patricio

C Aquiles

P Patricio

$\mathrm{V}$ María Isabel

M María Josefina

G Gabriela

L Chrystal

Glasinovic $\quad \mathrm{R} \quad$ Juan Carlos

Gloger

Godoy

Godoy

Goic
F Jaime

J Gonzalo

G Alejandro
Q Ronald

S Cecilia

L Miguel 
L

$\begin{array}{lll}\text { Labarca } & \text { B } & \text { Rodrigo } \\ \text { Ledermann } & \text { D } & \text { Walter } \\ \text { Letelier } & \text { S } & \text { Luz María } \\ \text { Liberman } & \text { G } & \text { Claudio } \\ \text { Lioi } & \text { C } & \text { Ximena } \\ \text { Lisboa } & \text { B } & \text { Carmen } \\ \text { Lissi } & \text { G } & \text { Eduardo } \\ \text { Lizana } & \text { S } & \text { Carlos } \\ \text { Lolas } & \text { S } & \text { Fernando } \\ \text { López } & \text { K } & \text { Francisco } \\ \text { Luco } & \text { F } & \text { Cristian } \\ \text { Luchsinger } & \text { F } & \text { Vivian }\end{array}$

\section{M}

Madrid

Maggi

Maggiolo

Maiz

Majlis

Manrique

Margozzini

Marshall

Martínez

Mateluna

Matus

Medina

Mella

Mericq

Meruane

Mezzano

Michaud

Millán

Miranda

Miranda

Molina

Monsalve

Moreno

Moreno

Mullins

Munizaga

Muñoz

Muzzo

\section{$\mathbf{N}$}

Neira

o

Ojeda

$\begin{array}{ll}\text { S } & \text { Ana María } \\ \text { C } & \text { Leonardo } \\ \text { G } & \text { Pedro } \\ \text { G } & \text { Alberto } \\ \text { L } & \text { Alejandro } \\ \text { E } & \text { Mónica } \\ \text { R } & \text { Juan } \\ \text { R } & \text { Guillermo } \\ \text { C } & \text { Virginia } \\ \text { G } & \text { Ester } \\ \text { C } & \text { Patricia } \\ \text { L } & \text { Ernesto } \\ \text { G } & \text { Iris } \\ \text { Q } & \text { Verónica } \\ \text { S } & \text { Jorge } \\ \text { A } & \text { Diego } \\ \text { Ch } & \text { Patricio } \\ \text { K } & \text { Teresa } \\ \text { M } & \text { Teresa } \\ \text { C } & \text { Marcelo } \\ \text { C } & \text { Ramiro } \\ \text { M } & \text { Verónica } \\ \text { B } & \text { Rodrigo } \\ \text { G } & \text { Manuel } \\ \text { L } & \text { Enrique } \\ \text { C } & \text { Fernando } \\ \text { N } & \text { Sergio } \\ \text { B } & \text { Santiago } \\ & \end{array}$

Q Oscar

S Dagoberto
Ojeda

Olmos

Olmos

Ortega

O'Ryan

Ossandón

Oviedo

Oyarzún

Oyonarte

P

Palestini

Palisson

Palma

Papapietro

Pardo

Paris

Parra

Pérez

Pérez

Pérez

Pérez

Perret

Pertuzé

Pino

Pinto

Pisano

Plubins

Poniachik

Prado

Prieto

Puga

Puschel

Q

Quezada

Quintana

Quiñones

\section{R}

Rabagliati

Raggio

Rappoport

Repetto

Rey

Reyes

Reyes

Reynolds

Ribalta
F José Manuel

C Alfonso

C Pablo

$\mathrm{P} \quad$ Rina

G Miguel

M Morelia

G Sofía

G Manuel

G Miguel

Q Mario

E Francis

C Renato

$\mathrm{V}$ Karin

G José

$\mathrm{M}$ Enrique

$\mathrm{R} \quad$ Claudio

P Gilberto

B Francisco

C Carlos

$\mathrm{H}$ María Angélica

P Cecilia

R Julio

Z Paulina

C Juan

O Raúl

M Luis

$\mathrm{T}$ Jaime

B Rodrigo

D Juan Carlos

P Julio

I Klaus

L Arnoldo

F Juan Carlos

$\mathrm{S} \quad$ Luis

\section{B Ricardo}

M Ximena

$\mathrm{S}$ Jaime

L Gabriela

N Severino

F Juan

O Viviana

$\mathrm{H}$ Enrique

$\mathrm{S}$ José 


\begin{tabular}{|c|c|c|c|c|c|}
\hline Ribalta & $\mathrm{L}$ & Gloria & $\mathbf{U}$ & & \\
\hline Rigotti & $\mathrm{R}$ & Attilio & Uauy & $\mathrm{D}$ & Ricardo \\
\hline Ríos & $\mathrm{L}$ & Ernesto & Ugarte & $\mathrm{P}$ & Francisca \\
\hline Ríoseco & St & Pedro & Ulloa & F & María Teresa \\
\hline Roa & $\mathrm{E}$ & Iván & Undurraga & S & María Soledad \\
\hline Roa & St. & Juan Carlos & Uribe & $\mathrm{J}$ & Pedro \\
\hline Rodríguez & $\mathrm{T}$ & Alberto & Uribe & $\mathrm{M}$ & Mario \\
\hline Rodríguez & $\mathrm{T}$ & Jorge & & & \\
\hline Rodríquez & Sch & Cristina & $\mathbf{V}$ & & \\
\hline Rodríguez & $\mathrm{D}$ & Juan Carlos & Vacarezza & Y & Ricardo \\
\hline Roessler & B & Emilio & Vásquez & M & Alicia \\
\hline Rollán & $\mathrm{R}$ & Antonio & Vaisman & W & Sergio \\
\hline Rosemblatt & S & Mario & Valdivia & C & Gonzalo \\
\hline Rosselot & $\mathrm{J}$ & Eduardo & Valenzuela & E & Jorge \\
\hline Rossi & $\mathrm{F}$ & Ricardo & Valenzuela & $\mathrm{Y}$ & Carlos \\
\hline Rozowski & $\mathrm{N}$ & Jaime & Valenzuela & M & Raúl \\
\hline & & & Vantman & B & David \\
\hline $\mathbf{S}$ & & & Vargas & $\mathrm{C}$ & Nelson \\
\hline Sáez & $\mathrm{H}$ & Enzo & Véjar & $\mathrm{P}$ & Claudia \\
\hline Saieh & A & Carlos & Véliz & $\mathrm{L}$ & Jesús \\
\hline Salas & del C & Cristián & Vergara & E & Mauricio \\
\hline Salvo & $\mathrm{C}$ & Karen & Vergara & E & Fernando \\
\hline Sánchez & $\mathrm{D}$ & Ignacio & Vicencio & A & María \\
\hline Santos & A & Manuel & Villarroel & D & Fernando \\
\hline Sapunar & Z & Jorge & Villaseca & D & Paulina \\
\hline Sarrá & C & Salvador & & & \\
\hline Sazunic & $\mathrm{Y}$ & Ivo & $\mathbf{W}$ & & \\
\hline Schiattino & $\mathrm{L}$ & Irene & Wageman & B & Rolando \\
\hline Segovia & D & Isabel & Wainstein & G & Eduardo \\
\hline Sepúlveda & $\mathrm{C}$ & Cecilia & Weitz & V & Juan Carlos \\
\hline Serón-Ferré & & María & Wistuba & 0 & Iván \\
\hline Silva & S & Juan Jorge & Wohllk & G & Nelson \\
\hline Silva & Z & Claudio & $\mathrm{Wu}$ & $\mathrm{H}$ & Elba \\
\hline Silva & C & Hugo & & & \\
\hline Silva & $\mathrm{O}$ & Juana & $\mathbf{Y}$ & & \\
\hline Soto & F & Rodrigo & Yáñez & $\mathrm{L}$ & Alfredo \\
\hline Spencer & 0 & Eugenio & Yazigi & I & Roberto \\
\hline $\mathbf{T}$ & & & $\mathbf{Z}$ & & \\
\hline Talesnik & $\mathrm{G}$ & Eduardo & Zagolin & B & Mónica \\
\hline Tapia & I & Patricia & Zapata & $\mathrm{L}$ & Rodrigo \\
\hline Téllez & $\mathrm{T}$ & Alvaro & Zilleruelo & B & Raúl \\
\hline Toche & $\mathrm{P}$ & Paola & & & \\
\hline Torres & $\mathrm{H}$ & Marisa & \multicolumn{3}{|c|}{ Comentarios Bibliográficos } \\
\hline Trucco & $\mathrm{A}$ & Olivia & Cruz-Coke & $\mathrm{M}$ & Ricardo \\
\hline Trucco & B & Marcelo & Marin & $\mathrm{L}$ & Pedro Paulo \\
\hline Turner & G & Eduardo & Meruane & $\mathrm{S}$ & Jorge \\
\hline
\end{tabular}

